\title{
Estudo do uso de Benzodiazepínicos em Instituição pública de Teresina-PI
}

\author{
Study of the use of Benzodiazepines in a public institution in Teresina-PI \\ Estudio del uso de Benzodiacepinas en una institución pública de Teresina-PI
}

Recebido: 16/12/2021 | Revisado: 22/12/2021 | Aceito: 24/12/2021 | Publicado: 05/01/2022

\author{
Gustavo Do Vale Batista \\ ORCID: https://orcid.org/0000-0002-7284-3563 \\ Centro Universitário UNINOVAFAPI, Brasil \\ E-mail:gugabatista95@gmail.com \\ Glaubert Damon da Silva Santos \\ ORCID: https://orcid.org/0000-0001-7901-8999 \\ Centro Universitário UNINOVAFAPI, Brasil \\ E-mail: gdamon9@hotmail.com \\ Williams Cardec da Silva \\ ORCID: https://orcid.org/0000-0002-7167-4876 \\ Centro Universitário UNINOVAFAPI, Brasil \\ E-mail: willcardec@hotmail.com
}

\begin{abstract}
Resumo
Benzodiazepínicos são os ansiolíticos mais utilizados para o tratamento de alguns problemas de saúde pulica, em especial por pacientes que fazem atendimento em CAPS, porém os mesmos podem apresentar efeitos perigosos quando tomados de maneira indevida. Com isso o trabalho objetivou objetivo avaliar o contexto brasileiro de disponibilidade e consumo de benzodiazepínicos, a partir do ponto de vista dos usuários do CAPS do município de Teresina-PI, do profissional de saúde e da dispensa desses medicamentos. A pesquisa foi realizada na CAPS do município de Teresina-PI no período de agosto e setembro de 2021. Todos os resultados foram respaldados através da aprovação pelo comitê de ética do centro universitário UNINOVAFAPI, as informações sendo recolhidas através de questionários enviados via email ou whatsapp. Como perfil observou-se uma maioria de mulheres na faixa de 51 a 62 anos, solteiras, $1^{\circ}$ grau completo e com outros 3 membros na residência. Quanto aos motivos de inicio do uso de medicamentos, a combinação de ansiedade, depressão e insônia foi observado em $66,2 \%$ dos pacientes. Houve a majoritariedade de consumo de clonazepam pelos pacientes dentre os benzodiazepínicos. Em um apanhado geral, a dosagem $2 \mathrm{mg}$ uma vez ao dia no período da noite no período da noite foi a posologia mais utilizada. Ainda foi observada a modificação do uso ou retirada do medicamento dos pacientes, essa medida sendo tomada muitas vezes pelo próprio usuário, sem recomendação medica.
\end{abstract}

Palavras-chave: Benzodiazepínico; Ansiolítico; Ansiedade.

\begin{abstract}
Benzodiazepines are the most used anxiolytics for the treatment of some public health problems, especially by patients who receive assistance in CAPS, but they can have dangerous effects when taken improperly. Thus, the study aimed to evaluate the Brazilian context of availability and consumption of benzodiazepines, from the point of view of users of CAPS in the city of Teresina-PI, health professionals and the dispensing of these drugs. The research was carried out at CAPS in the municipality of Teresina-PI between August and September 2021. All results were supported by approval by the ethics committee of the UNINOVAFAPI university center, the information being collected through questionnaires sent via email or whatsapp. As a profile, there was a majority of women aged 51 to 62 years, single, complete elementary school and with 3 other members in the household. As for the reasons for starting to use medication, the combination of anxiety, depression and insomnia was observed in $66.2 \%$ of patients. There was a majority of clonazepam consumption by patients among benzodiazepines. Overall, the dosage of $2 \mathrm{mg}$ once a day in the evening or at night was the most used dosage. Modification of the use or withdrawal of medication from patients was also observed, this measure being often taken by the user, without medical recommendation.
\end{abstract}

Keyword: Benzodiazepine; Anxiolytic; Anxiety.

\section{Resumen}

Las benzodiazepinas son los ansiolíticos más utilizados para el tratamiento de algunos problemas de salud pública, especialmente por pacientes que reciben asistencia en CAPS, pero pueden tener efectos peligrosos si se toman incorrectamente. Así, el estudio tuvo como objetivo evaluar el contexto brasileño de disponibilidad y consumo de benzodiacepinas, desde el punto de vista de los usuarios de CAPS en la ciudad de Teresina-PI, los profesionales de la salud y la dispensación de estos fármacos. La investigación se realizó en el CAPS de la ciudad de Teresina-PI entre 
agosto y septiembre de 2021. Todos los resultados fueron avalados por la aprobación del comité de ética del centro universitario UNINOVAFAPI, siendo recolectada la información a través de cuestionarios enviados vía correo electrónico o whatsapp. A modo de perfil, se observó una mayoría de mujeres entre 51 y 62 años, solteras, con educación primaria completa y con otros 3 miembros en el hogar. En cuanto a los motivos para empezar a utilizar la medicación, la combinación de ansiedad, depresión e insomnio se observó en el 66,2\% de los pacientes. Hubo una mayoría de consumo de clonazepam por parte de los pacientes entre las benzodiazepinas. En general, la dosis de $2 \mathrm{mg}$ una vez al día por la tarde o por la noche fue la dosis más utilizada. También se observó modificación del uso o retiro de medicación de los pacientes, medida que a menudo es tomada por el usuario, sin recomendación médica.

Palabras clave: Benzodiazepina; Ansiolítico; Ansiedad.

\section{Introdução}

Os Benzodiazepínicos (BDZs) são drogas com atividade ansiolítica que começaram a ser utilizadas na década de 60 . O Clordiazepóxido foi o primeiro BDZ lançado no mercado (1960), cinco anos após a descoberta de seus efeitos ansiolíticos, hipnóticos e miorrelaxantes. Além da elevada eficácia terapêutica, os BDZs apresentaram baixos riscos de intoxicação e dependência, fatores estes que propiciaram uma rápida aderência da classe médica a esses medicamentos (Laforgue et al., 2020; Muzaale et al., 2020).

Nos anos posteriores foram observados os primeiros casos de uso abusivo, além de desenvolvimento de tolerância, de síndrome de abstinência e de dependência pelos usuários crônicos de BDZs. Tais evidências modificaram a postura da sociedade em relação aos BDZs que, do auge do entusiasmo nos anos 70, passou à restrição do uso a partir da década seguinte. Nos Estados Unidos, por exemplo, o uso destes medicamentos pela população chegou a atingir 11,1\% em 1979, diminuindo para 8,3\%, em 1990 (Deka et al., 2018; Luta et al., 2020).

O uso prolongado de BDZs, ultrapassando períodos de 4 a 6 semanas pode levar ao desenvolvimento de tolerância, abstinência e dependência. A possibilidade de desenvolvimento de dependência deve sempre ser considerada, principalmente na vigência de fatores de risco para a mesma, tais como uso em mulheres idosas, em poli usuários de drogas, para alívio de estresse, de doenças psiquiátricas e distúrbios do sono. Também é comum a observação de overdose de BDZs entre as tentativas de suicídio, associados ou não a outras substâncias (Hernandez et al., 2018).

Os efeitos adversos comuns no uso prolongado são os déficits cognitivos (perda de atenção e dificuldade de fixação), fraqueza, náuseas, vômitos, dores abdominais, diarreia, dores articulares e torácicas, incontinência urinária, desequilíbrio, pesadelos, taquicardia, alucinações, hostilidade e alteração do comportamento, que tendem a se instalar no curso da utilização desses medicamentos. Há risco aumentado de quedas, problemas respiratórios em idosos (Airagnes et al., 2019).

Órgãos internacionais, como a OMS (Organização Mundial da Saúde) e o INCB (Internacional Narcotics Control Board), têm alertado sobre o uso indiscriminado e o insuficiente controle de medicamentos psicotrópicos nos países em desenvolvimento. No Brasil, esse alerta foi reforçado por estudos das décadas de 80 e 90 que mostraram uma grave realidade relacionada ao uso de benzodiazepínicos. No primeiro levantamento domiciliar nacional realizado em 2001, 3,3\% dos entrevistados (entre 12 e 65 anos) afirmaram uso de benzodiazepínicos sem receita médica. Em um outro levantamento, com estudantes da rede pública de ensino de dez capitais brasileiras, 5,8\% dos entrevistados afirmaram já ter feito uso de ansiolíticos sem prescrição (Victorri-Vigneau et al., 2021).

No ano de 1999, foi realizado estudo em dois municípios brasileiros, no qual foi analisado um universo de 108.215 notificações e receitas especiais retidas em farmácias, drogarias, postos de saúde, hospitais. Esse estudo indicou descuido no preenchimento das notificações e receitas especiais e, inclusive, indícios de falsificações, na forma de prescrições por médicos falecidos e notificações com numeração oficial repetida. Essa realidade indica a necessidade de uma ampla revisão no atual sistema de controle dessas substâncias, bem como do papel dos profissionais de saúde nesse sistema (Osler et al., 2018). 
Para subsidiar essa discussão são necessários estudos que busquem não apenas quantificar, mas compreender as crenças e valores que embasam as atitudes dos profissionais de saúde e dos usuários de BDZ, devido o alto índice de usuários desses medicamentos (Wang et al., 2017).

Dessa forma, este estudo teve como objetivo avaliar o contexto brasileiro de disponibilidade e consumo de benzodiazepínicos, a partir do ponto de vista dos usuários dos Centros de Atenção Psicossocial do município de Teresina-PI e dos profissionais de saúde envolvidos no sistema de prescrição e dispensação desses medicamentos. Observando em especial qual dos BDZ são os mais utilizados e o perfil dos seus usuários.

\section{Metodologia}

Este estudo se caracteriza como uma pesquisa transversal, descritiva e exploratória com abordagem quantitativa (Gil, 2008; de Carvalho, 2021), a pesquisa descritiva visa descrever as características de determinadas populações ou fenômenos. Uma de suas peculiaridades está na utilização de técnicas padronizadas de coleta de dados, tais como o questionário e a observação sistemática.

Bloomfield e Fisher (2019) referem também que a pesquisa quantitativa reúne evidências empíricas e são números que resultam de algum tipo de medição formal que são analisados através de procedimentos estatísticos. Ressalta também que esse tipo de pesquisa não deve ser usado para responder questão ética ou moral.

A pesquisa foi realizada no Centro de Atenção Psicossocial do município de Teresina-PI, com coleta realizada no período de agosto e setembro de 2021.0 estudo foi submetido e aprovado pelo comitê de ética e pesquisa do Centro Universitário UNINOVAFAPI e submetido à plataforma Brasil, após essa aprovação iniciou-se a busca pelos participantes. Os pacientes que concordaram em participar da pesquisa receberam um Termo de Consentimento Livre e Esclarecido via email ou whatsapp, após o a assinatura dos mesmo os questionários para a coleta dados foram enviados.

A amostra populacional foi calculada através da formula: $\mathrm{n}=\left(\mathrm{z}^{2} \cdot 0,25 \cdot \mathrm{N}\right) /\left(\mathrm{E}^{2}(\mathrm{~N}-1)+\mathrm{z}^{2} \cdot 0,25\right)=$ $\left(1,96^{2} \cdot 0,25 \cdot 662\right) /\left(0,07^{2} \cdot 661+1,96^{2} \cdot 0,25\right)=151$, na qual, z é o valor crítico, E a margem de erro e $\mathrm{N}$ o tamanho da população, considerando o grau de confiança de $95 \%(\mathrm{z}=1,96)$, margem de erro $\mathrm{E}=7 \%$ e $\mathrm{N}=662$. Os participantes foram selecionados através de critérios de inclusão, usuários de benzodiazepínicos de forma prolongada, e exclusão, pacientes que não tinham capacidade de responder os questionários.

Os resultados foram organizados através do excel e analisados por SPSS utilizando a analise de qui-quadrado. Esses valores foram expressos por meio de tabelas e figuras.

\section{Resultados e Discussão}

O estudo teve sua coleta de dados respaldada através da aprovação pelo comitê de ética e autorização pela plataforma Brasil, para estudos com humanos, além desses documentos, os responsáveis pela pesquisa assinaram um termo de comprometimento com o estudo e sigilo de todas as informações pessoais coletada.

Para orientação dos participantes, todos assinaram ao TCLE, sendo tendo seus pontos explicados para que os voluntários tivessem completo conhecimento do projeto que estavam participando. Como mencionado, os dados foram obtidos através de um questionário realizado de forma remota através do envio do questionário via e-mail ou whatsapp.

A pesquisa contou com um total de 151 participantes com perfis sociais e econômicos distintos. Foram avaliados destes os motivos para procura de ajuda medica, medicamentos utilizados, dosagem e até sintomas que os mesmos sentem. 


\subsection{Perfil socioeconômico}

Com um percentual de 60,3\% de mulheres e 39,7\% homens, com idades variando de 18 à 62 anos, a maior parte deles entre 51 1- 62 anos (35,8\%). Com relação ao estado civil, 57\% dos participantes são solteiros, 27,2\% casados, 9,3\% divorciados e 6,6\% viúvos (Figura 1).

Figura 1. Estado civil dos participantes.

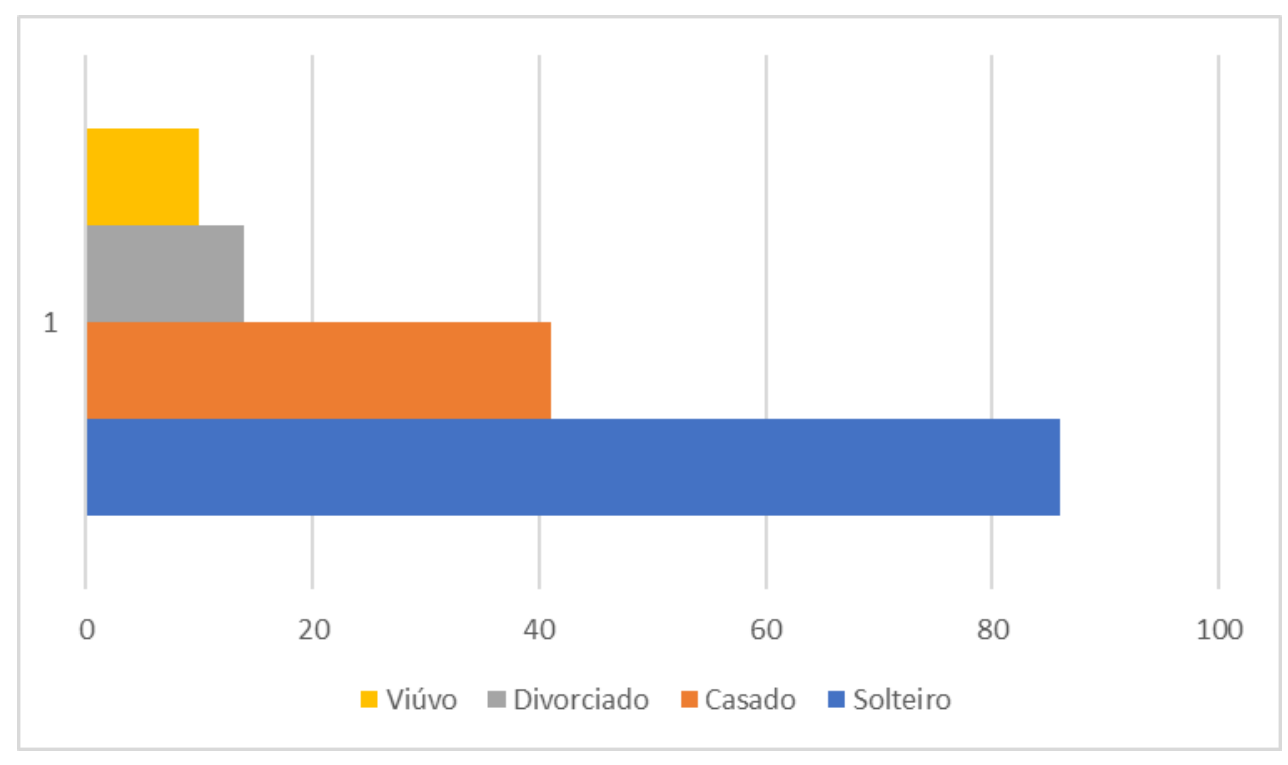

Fonte: Dados da pesquisa.

A renda mensal da maioria dos participantes é de até um salário mínimo (74,2\%), refletido principalmente pela escolaridade dos mesmos, em que a maioria tem até o $1^{\circ}$ grau completo $(49,7 \%)$ e $10,6 \%$ nunca chegou a frequentar escola. No aspecto educacional, $32,5 \%$ completaram o $2^{\circ}$ grau, $0,7 \%$ o $3^{\circ}$ graus e $6,6 \%$ o ensino superior.

Os participantes ainda relataram a quantidade de pessoas que residiam junto com eles, que podia ir de 0-9 pessoas. A maioria dos participantes moravam com outras três pessoas $(26,5 \%)$, com os menores percentuais sendo de zero e sete pessoas, com $0,7 \%$ para cada um (Tabela 1 ).

Conforme confirmado pela literatura, a escolaridade é um fator explicativo correlacionado ao conhecimento sobre o tratamento. Esse fator pode estar relacionado ao nível de conhecimento do paciente sobre a doença. Surpreendentemente, ao contrário da literatura existente, idade não foi associada a pior conhecimento das indicações de medicamentos (Jakobsen et al., 2018; Vozoris et al., 2019; Laforgue et al., 2020).

O intervalo de tempo entre o preenchimento da receita médica e a coleta de dados pelo médico pode ter afetado as taxas de conhecimento mais baixas. Em contrapartida, no momento do recrutamento, os pacientes foram informados de que seriam contatados especificamente para um estudo sobre seus medicamentos BZD e solicitados a trazer suas prescrições no momento da ligação, o que sugere que as taxas encontradas podem estar superestimadas (Bernard et al., 2019). 
Tabela 1. Número de residentes no local.

\begin{tabular}{cc}
\hline Número de pessoas & Frequência \\
\hline Zero & 1 \\
Um & 8 \\
Dois & 33 \\
Três & 40 \\
Quatro & 30 \\
Cinco & 19 \\
Seis & 11 \\
Sete & 1 \\
Oito & 6 \\
Nove & 2 \\
\hline
\end{tabular}

Fonte: Dados da pesquisa.

\subsection{Aspectos clínico}

Foi avaliado a origem da prescrição medicamentosa para os participantes, de todos apenas um obteve por meio de clinico geral, enquanto os outros $150(99,3 \%)$ por orientação de psiquiatra. Dos motivos para tal feito, houve uma variação entre ansiedade, depressão. Insônia, epilepsia, crise de pânico e associação dos mesmos (Tabela 2), sendo a combinação de ansiedade, depressão e insônia a mais predominante, a mesma foi relatada por 66,2\% dos participantes.

Uma possível fonte de viés subjacente à subestimação é a escolha de enfocar as questões psiquiátricas atuais, deixando assim de relatar os fatores subjacentes essenciais. Por exemplo, a ansiedade pode ser um sintoma de transtornos psiquiátricos como transtorno depressivo maior, transtorno de ansiedade generalizada, enquanto isso, o BZD trata os sintomas e não as etiologias (Herrera-Gómez et al., 2018; Sun et al., 2019; Torres-Bondia et al., 2020).

Na pesquisa proposta por Sonnenberg et al (2012) o uso de benzodiazepínicos permaneceu estável, além disso, o uso a longo prazo permaneceu alto, apesar das recomendações nas diretrizes para manter o tratamento curto, no entanto, em comparação com o aumento do uso de antidepressivos nesse período, a estabilidade no uso de benzodiazepínicos pode ser considerada um achado relativamente positivo. Uma tendência de preferência foi encontrada para benzodiazepínicos de curta duração sem metabólitos ativos farmacológicos.

De acordo com a literatura, ser mulher, ter uma ou mais doenças crônicas, depressão, problemas de sono e uso de antidepressivo permaneceram associados ao uso de benzodiazepínicos nas análises multivariadas e foram considerados como explicadores da associação com os demais fatores de risco (Victorri-Vigneau et al., 2019).

Tabela 2. Motivos para utilização dos medicamentos.

\begin{tabular}{cc}
\hline Motivo & Frequência \\
\hline Ansiedade & 1 \\
Ansiedade, depressão & 2 \\
Ansiedade, insônia & 15 \\
Ansiedade, depressão, insônia & 100 \\
Ansiedade, depressão, insônia, epilepsia & 19 \\
Insônia & 5 \\
Insônia, crise de pânico & 1 \\
Depressão & 1 \\
Depressão e insônia & 7 \\
\hline
\end{tabular}

Fonte: Dados da pesquisa.

Os medicamentos usados variaram entre quatro, bromezepam, clonazepam, Diazepam e Rivotril, os mesmos podiam apresentar diversas dosagens e formula farmacêutica, podendo ser em forma de comprimido ou em gotas. Dentre os medicamentos utilizados, o clonazepam foi o mais receitado para os pacientes, com 80,1\% dos voluntários fazendo o seu uso durante o tratamento (Figura 2). 
Figura 2. Tipo de medicamento consumido.

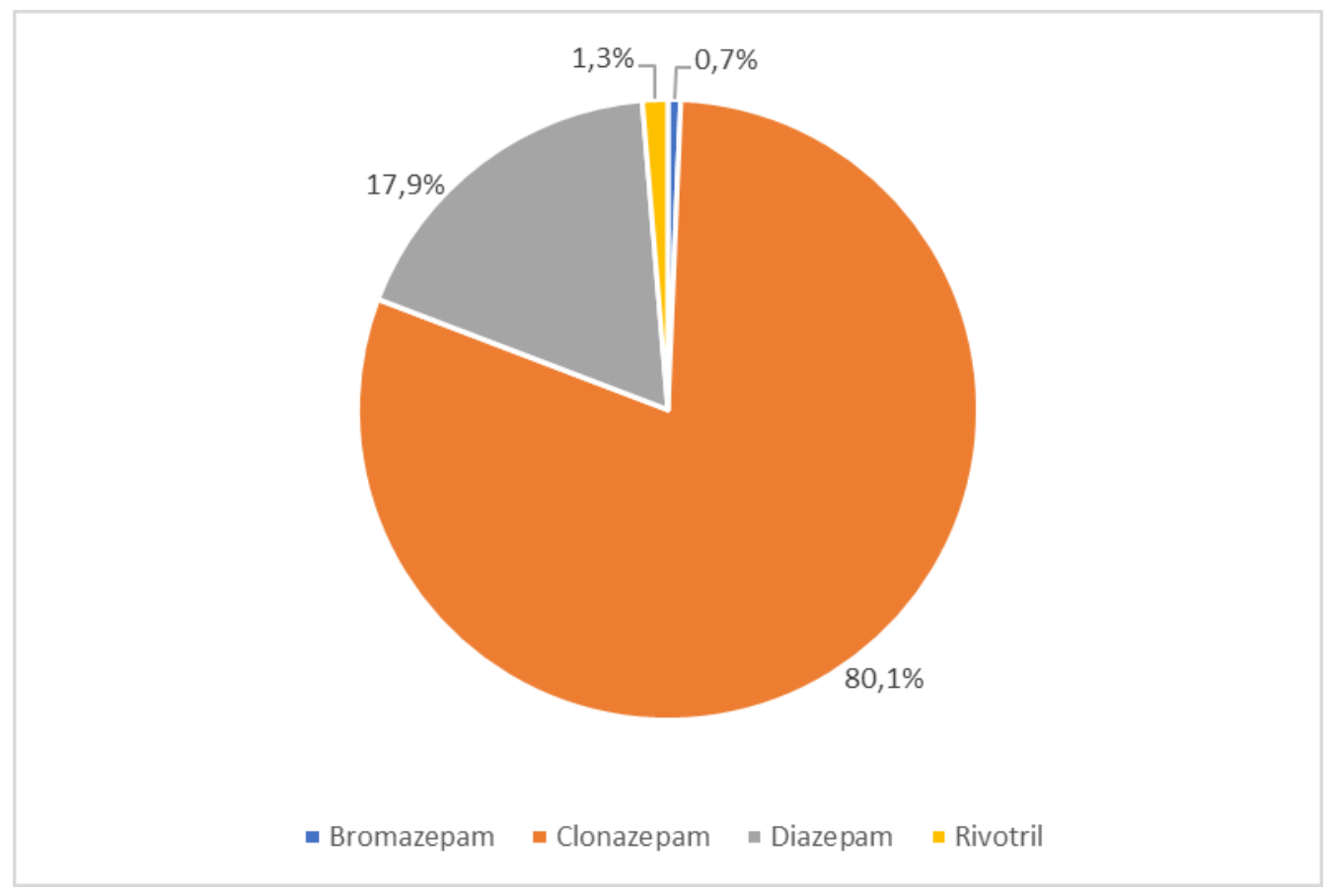

Fonte: Dados da pesquisa.

As dosagens diárias consumidas foram analisadas, porém não foi feita de forma especifica por medicamento, apenas o quantitativo ingerido pelos voluntario. Foi observado uma grande variabilidade das doses (Tabela 3), com o maior percentual sendo de $2 \mathrm{mg}$ uma vez ao dia $(50,3 \%)$, seguido de $4 \mathrm{mg}(6,6 \%)$ e $5 \mathrm{mg}(6,6 \%)$ uma vez ao dia. As demais dosagens variaram de 9 à 1 participante.

De acordo com o estudo de Fride et al (2015) indivíduos que começaram com alprazolam também tiveram um risco maior de acabar como usuários de alto BZD em comparação com aqueles que começaram com diazepam. Deve-se notar, entretanto, que embora a taxa de risco estimada fosse alta, isso preocupava poucas pessoas. Apenas 187 indivíduos iniciaram o uso de alprazolam e 12 destes acabaram como usuários excessivos. Os médicos são alertados contra a prescrição de alprazolam, pois é conhecido por ser especialmente viciante e, portanto, nossa análise confirma um conhecimento bem estabelecido

No estudo efetuado por Marti (2020) o caso de depressão, ansiedade e problemas de sono, o uso de benzodiazepínicos manteve-se elevado, embora seja recomendado apenas para uso em curto prazo ou para suporte nas primeiras semanas de tratamento com antidepressivos ou psicoterapia. 
Research, Society and Development, v. 11, n. 1, e21611124839, 2022

(CC BY 4.0) | ISSN 2525-3409 | DOI: http://dx.doi.org/10.33448/rsd-v11i1.24839

Tabela 3. Dosagens ingeridas

\begin{tabular}{|c|c|}
\hline Dosagem & Frequência \\
\hline $0,5 \mathrm{mg} 1 \mathrm{x}$ ao dia & 1 \\
\hline $1 \mathrm{mg} 1 \mathrm{x}$ ao dia & 5 \\
\hline $2 \mathrm{mg} 1 \mathrm{x}$ ao dia & 76 \\
\hline $2 \mathrm{mg} 2 \mathrm{x}$ ao dia & 9 \\
\hline $2 \mathrm{mg} 3 \mathrm{x}$ ao dia & 2 \\
\hline 3 gotas ao dia & 3 \\
\hline $3 \mathrm{mg} 1 \mathrm{x}$ ao dia & 2 \\
\hline $4 \mathrm{mg} 1 \mathrm{x}$ ao dia & 10 \\
\hline $4 \mathrm{mg} 2 \mathrm{x}$ ao dia & 2 \\
\hline 5-8 gotas ao dia & 1 \\
\hline 5 gotas $2 \mathrm{x}$ ao dia & 1 \\
\hline $5 \mathrm{mg} 1 \mathrm{x}$ ao dia & 10 \\
\hline $5 \mathrm{mg} 2 \mathrm{x}$ ao dia & 2 \\
\hline $5 \mathrm{mg} 3 \mathrm{x}$ ao dia & 5 \\
\hline 6 gotas ao dia & 1 \\
\hline $6 \mathrm{mg} 1 \mathrm{x}$ ao dia & 3 \\
\hline $6 \mathrm{mg} 2 \mathrm{x}$ ao dia & 1 \\
\hline 8 gotas ao dia & 1 \\
\hline $8 \mathrm{mg} 1 \mathrm{x}$ ao dia & 1 \\
\hline 9 gotas ao dia & 1 \\
\hline 10 gotas ao dia & 2 \\
\hline $10 \mathrm{mg} 1 \mathrm{x}$ ao dia & 7 \\
\hline $10 \mathrm{mg} 2 \mathrm{x}$ ao dia & 1 \\
\hline $15-20$ gotas ao dia & 1 \\
\hline $20 \mathrm{mg} 1 \mathrm{x}$ ao dia & 2 \\
\hline $15 \mathrm{mg} \mathrm{C}$ e $2 \mathrm{mg} \mathrm{D}$ & 1 \\
\hline
\end{tabular}

Fonte: Dados da pesquisa.

Os horários da administração também foram observados, já que alguns dos pacientes precisavam da medicação mais de uma vez ao dia, esses podiam variar entre manhã (8-12h), tarde (13-18h) e noite (19-00h) (Figura 3). Dentre os horários a administração uma no período da noite foi a que observou maior percentual de 62,6\%, alguns dos participantes não determinaram o horário especifico ou informaram que ficava à critério do mesmo o horário, esses foram classificados como indeterminados. 
Figura 3. Consumo do medicamento de acordo com o horário.

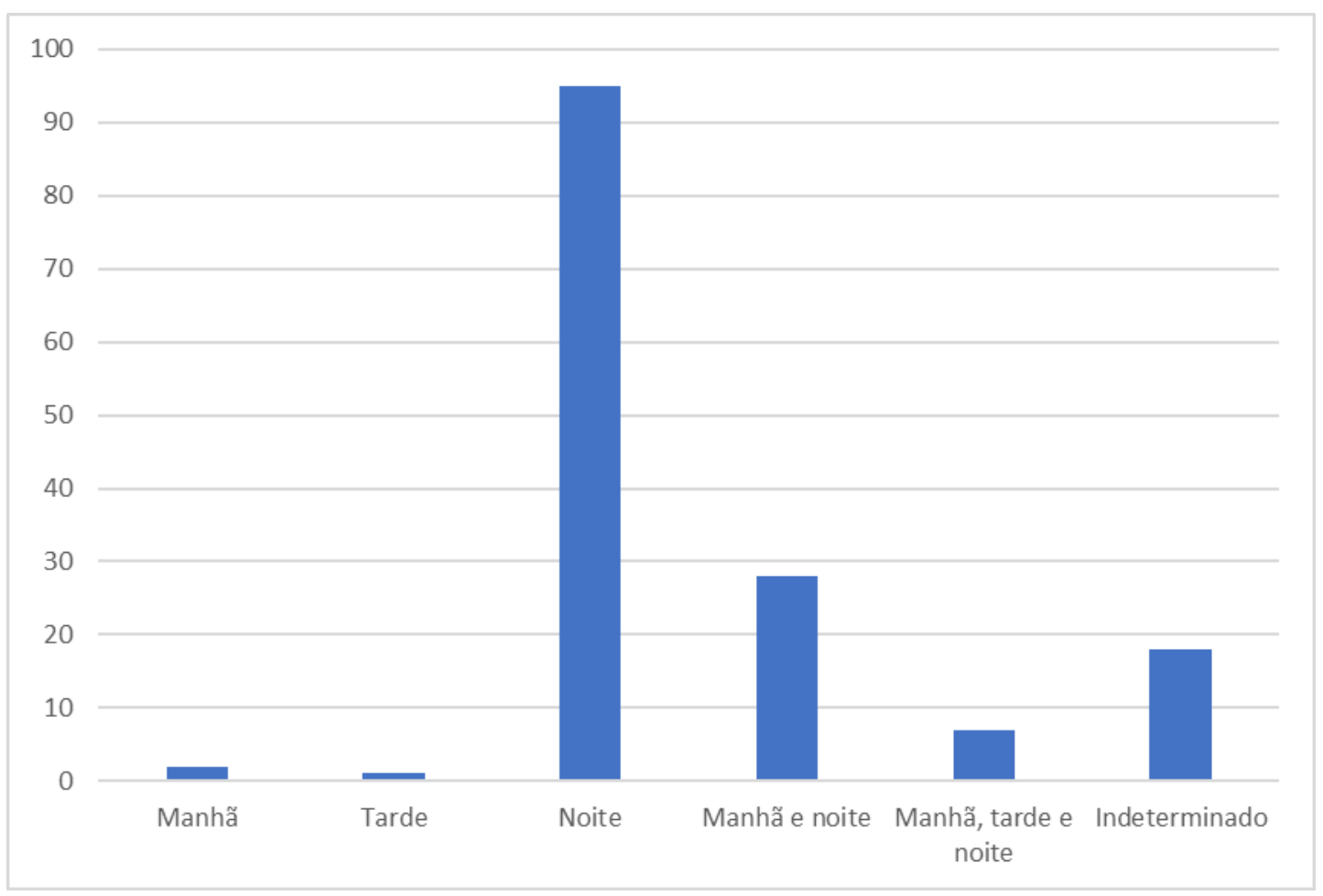

Fonte: Dados da pesquisa.

\subsection{Resultados observados devido a medicação}

Quase a totalidade dos pacientes (96\%) demonstraram melhora após o inicio do uso dos medicamentos. Foi observada também o período de uso dos mesmos, tanto em quantos dias quando a intermitência deles, esses podendo ser quando necessário, 60 dias, 120 dias ou acima de 120 dias, de forma continua ou intermitente.

Quanto aos dias 98\% informaram que sua medicação tem uma posologia acima de 120 dias, enquanto os demais apresentaram apenas um percentual de $0,7 \%$ cada. Com relação a continuidade, $96 \%$ apresentam uma utilização continua, enquanto apenas $4 \%$ podem realizar o mesmo de forma intermitente.

A combinação do uso de benzodiazepínicos com o uso de álcool é importante devido à possibilidade de sedação excessiva e distúrbios do humor. Nos usuários de álcool, o uso de benzodiazepínicos foi menor do que na amostra completa, mas alguns entrevistados combinaram álcool com benzodiazepínicos, o que pode levar a problemas de saúde importantes, por exemplo, maior risco de quedas, problemas de memória e acidentes de trânsito, principalmente quando esse grupo envelhece (Airagnes et al., 2019; Luta et al., 2020).

Juntamente com isso, $61,9 \%$ dos participantes informaram que a sua dosagem foi alterada em algum momento durante o processo, porém destes, apenas $63,4 \%$ fizeram esta modificação devido orientação médica, os demais 36,6\% alteraram por conta própria, podendo prejudicar o seu tratamento (Figura 4). 
Figura 4. Alteração da posologia do tratamento.

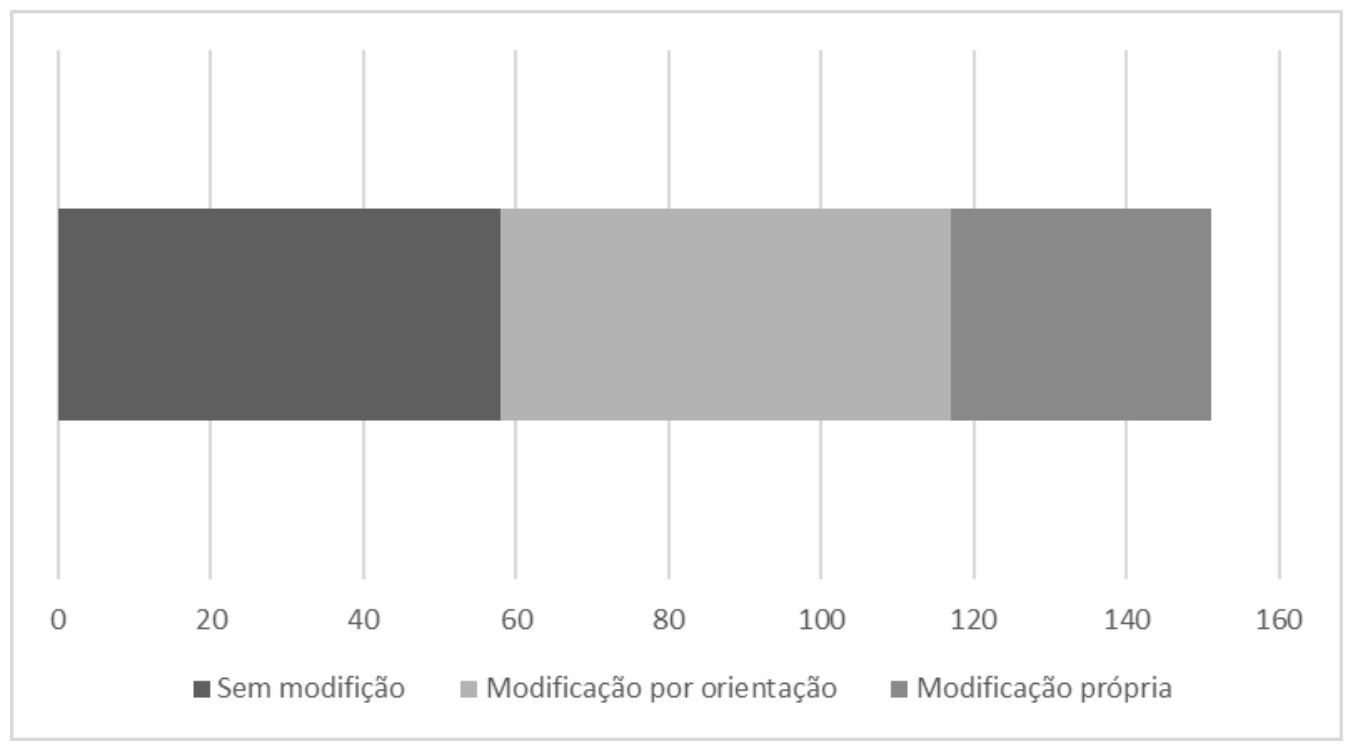

Fonte: Dados da pesquisa.

Assim como a parada do uso dos medicamentos também foi observado, em que 26,5\% já o fizeram, porém apenas $15,4 \%$ deles foi por pedido do psiquiatra, e de forma ainda mais perigosa $66,7 \%$ deles retiraram o medicamento de forma súbita, com uma grande possibilidade de gerar efeitos colaterais (Figura 5).

Figura 5. Parada do uso do medicamento.

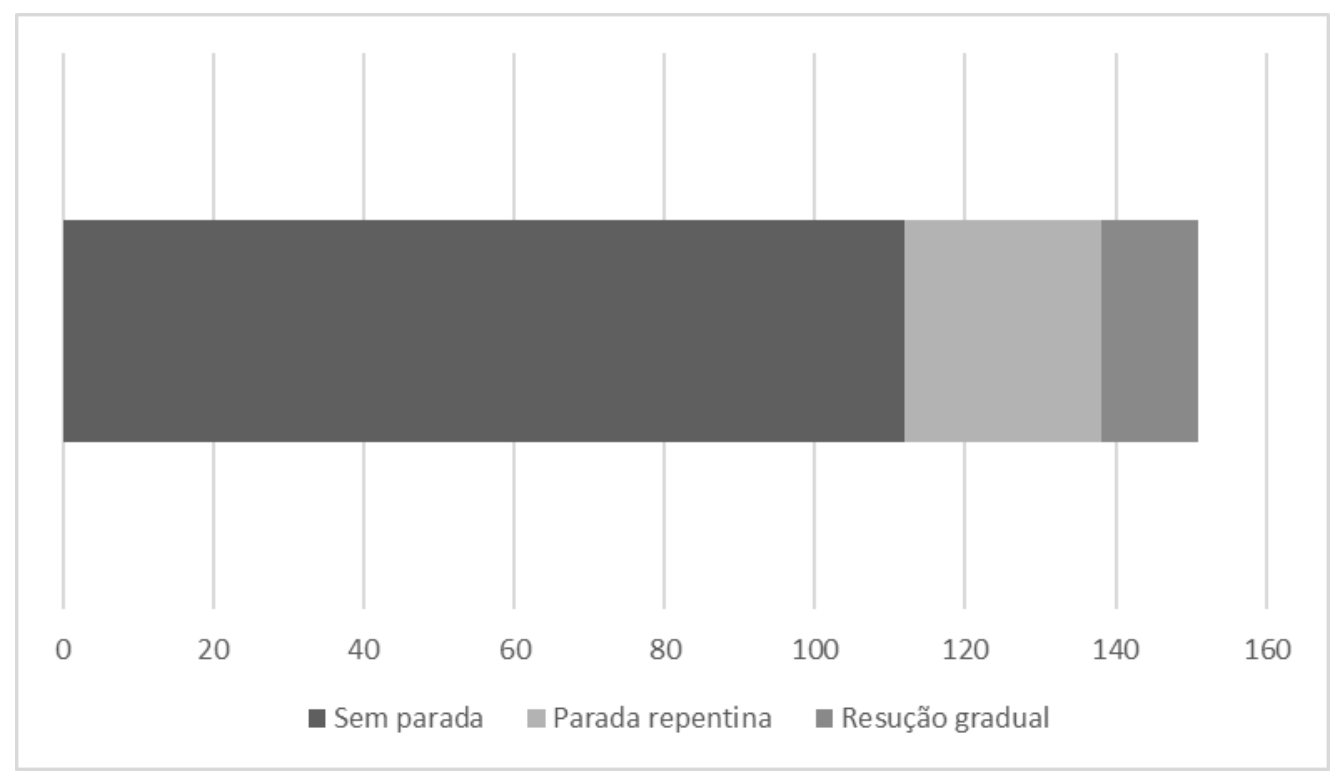

Fonte: Dados da pesquisa.

O último fator analisado foram os possíveis efeito observados devido a essa ausência do medicamente, esses sintomas variando de ansiedade, insônia, agitação, pânico, irritabilidade, tremor, dificuldade de respirar, dificuldade de concentração, conversar sozinho, depressão, epilepsia, nervosismo, medo, tristeza, vozes e dor no corpo, podendo de ser de forma isolada ou combinada. 
Nessa visão a junção de ansiedade, agitação e insônia foi a sintomatologia mais observada por abstinência do medicamento, com cerca de 4,64\% dos participantes apresentando o mesmo. Importante ressaltar que $2 \%$ dos voluntario que fizeram a parada não apresentaram nenhum sintoma após esse corte.

Embora diferenças possam ser encontradas dependendo se clínicos gerais ou especialistas são os prescritores, esse problema global começou há mais de 20 anos. À medida que a idade aumenta, as pessoas ficam mais sensíveis a uma terapia de benzodiazepina mais longa e a doses mais altas, embora os distúrbios permaneçam os mesmos para as populações mais jovens (Victorri-Vigneau et al., 2021; Hernandez et al., 2018).

Além disso, os pacientes também podiam tomar mais do medicamento do que o recomendado, sem informar aos médicos que o tratavam. Portanto, são necessárias medidas para controlar a quantidade prescrita (Luta et al., 2020).

\section{Conclusão}

Em conclusão, a pesquisa realizada através de questionário no Centro de Atenção Psicossocial do município de Teresina-PI enviado aos participantes demonstrou efeitos significativos sobre o conhecimento da medicação, crenças e percepção de risco em uma coorte de usuários de benzodiazepínicos.

Ao mudar o conhecimento e aumentar o risco percebido, as informações sobre medicamentos direcionadas ao consumidor despertaram o desejo de muitas pesquisas de discutir a segurança dos medicamentos com seus profissionais de saúde. Os resultados de um ensaio clínico randomizado poderão demostrar futuramente se essas mudanças provocadas pela intervenção educacional são suficientes para resultar na descontinuação de prescrições inadequadas.

\section{Referências}

Airagnes, G., Lemogne, C., Renuy, A., Goldberg, M., Hoertel, N., Roquelaure, Y., \& Zins, M. (2019). Prevalence of prescribed benzodiazepine long-term use in the French general population according to sociodemographic and clinical factors: findings from the CONSTANCES cohort. BMC Public Health, 19(1), 19.

Bernard, M. M. T., Luc, M., Carrier, J. D., Fournier, L., Duhoux, A., Côté, E., \& Roberge, P. (2018). Patterns of benzodiazepines use in primary care adults with anxiety disorders. Heliyon, 4(7), e00688.

Bloomfield, J., \& Fisher, M. J. (2019). Quantitative research design. Journal of the Australasian Rehabilitation Nurses Association, 22(2), 27-30.

de Carvalho, M. C. M. (2021). Construindo o saber: Metodologia científica-Fundamentos e técnicas. Papirus Editora.

Deka, R., Bryan, C. J., LaFleur, J., Oderda, G., Atherton, A., \& Stevens, V. (2018). Benzodiazepines, health care utilization, and suicidal behavior in veterans with posttraumatic stress disorder. The Journal of clinical psychiatry, 79(6), 0-0.

Fride Tvete, I., Bjørner, T., \& Skomedal, T. (2015). Risk factors for excessive benzodiazepine use in a working age population: a nationwide 5-year survey in Norway. Scandinavian journal of primary health care, 33(4), 252-259.

Gil, A. C. (2008). Métodos e técnicas de pesquisa social. (6a ed.), Ediitora Atlas SA.

Hernandez, I., He, M., \& Zhang, Y. (2018). Comparing state, regional, and local variation in concurrent opioid and benzodiazepine use. Drug and alcohol dependence, 191, 141-144.

Herrera-Gómez, F., Gutierrez-Abejón, E., Criado-Espegel, P., \& Álvarez, F. J. (2018). The problem of benzodiazepine use and its extent in the driver population: a population-based registry study. Frontiers in pharmacology, 9, 408.

Jakobsen, L. S., Georgiadis, S., Nielsen, B. F., Bokkers, B. G., Boriani, E., Duedahl-Olesen, L., \& Pires, S. M. (2018). Probabilistic approach for assessing cancer risk due to benzo [a] pyrene in barbecued meat: Informing advice for population groups. PloS one, 13(11), e0207032.

Laforgue, E. J., Jobert, A., Rousselet, M., Grall-Bronnec, M., FAN network, Jolliet, P., \& Victorri-Vigneau, C. (2020). Do older people know why they take benzodiazepines? A national French cross-sectional survey of long-term consumers. International journal of geriatric psychiatry, 35 (8), 870-876.

Luta, X., Bagnoud, C., Lambiris, M., Decollogny, A., Eggli, Y., Le Pogam, M. A., \& Marti, J. (2020). Patterns of benzodiazepine prescription among older adults in Switzerland: a cross-sectional analysis of claims data. BMJ open, 10(1), e031156. 
Research, Society and Development, v. 11, n. 1, e21611124839, 2022

(CC BY 4.0) | ISSN 2525-3409 | DOI: http://dx.doi.org/10.33448/rsd-v11i1.24839

Muzaale, A. D., Daubresse, M., Bae, S., Chu, N. M., Lentine, K. L., Segev, D. L., \& McAdams-DeMarco, M. (2020). Benzodiazepines, codispensed opioids, and mortality among patients initiating long-term in-center hemodialysis. Clinical journal of the American Society of Nephrology, 15(6), 794-804.

Osler, M., \& Jørgensen, M. B. (2020). Associations of benzodiazepines, Z-drugs, and other anxiolytics with subsequent dementia in patients with affective disorders: a nationwide cohort and nested case-control study. American Journal of Psychiatry, 177(6), 497-505.

Sonnenberg, C. M., Bierman, E. J., Deeg, D. J., Comijs, H. C., Van Tilburg, W., \& Beekman, A. T. (2012). Ten-year trends in benzodiazepine use in the Dutch population. Social psychiatry and psychiatric epidemiology, 47(2), 293-301.

Sun, G. Q., Zhang, L., Zhang, L. N., Wu, Z., \& Hu, D. F. (2019). Benzodiazepines or related drugs and risk of pneumonia: A systematic review and meta-analysis. International journal of geriatric psychiatry, 34(4), 513-521.

Torres-Bondia, F., de Batlle, J., Galván, L., Buti, M., Barbé, F., \& Piñol-Ripoll, G. (2020). Trends in the consumption rates of benzodiazepines and benzodiazepine-related drugs in the health region of Lleida from 2002 to 2015. BMC Public Health, 20, 1-9.

Victorri-Vigneau, C., Laforgue, E. J., Grall-Bronnec, M., Guillou-Landreat, M., Rousselet, M., Guerlais, M., \& Jolliet, P. (2021). Are seniors dependent on benzodiazepines? A national clinical survey of substance use disorder. Clinical Pharmacology \& Therapeutics, 109(2), 528-535.

Vozoris, N. T. (2019). Benzodiazepine and opioid co-usage in the US population, 1999-2014: An exploratory analysis. Sleep, 42(4), zsy264.

Wang, M. T., Wang, Y. H., Chang, H. A., Tsai, C. L., Yang, Y. S., Lin, C. W., \& Hsu, Y. J. (2017). Benzodiazepine and Z-drug use and risk of pneumonia in patients with chronic kidney disease: a population-based nested case-control study. PloS one, 12(7), e0179472. 